\title{
Communication \\ Compact Photonic Crystal Polarization Beam Splitter Based on the Self-Collimation Effect
}

\author{
Geyu Tang ${ }^{1}$, Huamao Huang ${ }^{1}$, Yuqi Liu ${ }^{1,2}$ and Hong Wang ${ }^{1,2, *(\mathbb{B}}$ \\ 1 Engineering Research Center for Optoelectronics of Guangdong Province, \\ School of Physics and Optoelectronics, South China University of Technology, Guangzhou 510640, China; \\ 201820127910@mail.scut.edu.cn (G.T.); schhm@scut.edu.cn (H.H.); phyqliu@mail.scut.edu.cn (Y.L.) \\ 2 Guangdong Provincial Engineering Center for Wide Bandgap Semiconductor Chips and Application, \\ Zhongshan Institute of Modern Industrial Technology, South China University of Technology, \\ Zhongshan 528437, China \\ * Correspondence: phhwang@scut.edu.cn; Tel.: +86-20-87111557
}

check for updates

Citation: Tang, G.; Huang, H.; Liu, Y.; Wang, H. Compact Photonic Crystal Polarization Beam Splitter Based on the Self-Collimation Effect. Photonics 2021, 8, 198. https://doi.org/ $10.3390 /$ photonics 8060198

Received: 7 May 2021

Accepted: 3 June 2021

Published: 4 June 2021

Publisher's Note: MDPI stays neutral with regard to jurisdictional claims in published maps and institutional affiliations.

Copyright: (c) 2021 by the authors. Licensee MDPI, Basel, Switzerland. This article is an open access article distributed under the terms and conditions of the Creative Commons Attribution (CC BY) license (https:/ / creativecommons.org/licenses/by/ $4.0 /)$

\begin{abstract}
We propose a new compact polarization beam splitter based on the self-collimation effect of two-dimensional photonic crystals and photonic bandgap characteristics. The device is composed of a rectangular air holes-based polarization beam splitting structure and circular air holes-based selfcollimating structure. By inserting the polarization beam splitting structure into the self-collimating structure, the TE and TM polarized lights are orthogonally separated at their junction. When the number of rows in the hypotenuse of the inserted rectangular holes is 5, the transmittance of TE polarized light at $1550 \mathrm{~nm}$ is $95.4 \%$ and the corresponding polarization extinction ratio is $23 \mathrm{~dB}$; on the other hand, the transmittance of TM polarized light is $88.5 \%$ and the corresponding polarization extinction ratio is $37 \mathrm{~dB}$. For TE and TM polarized lights covering a $100 \mathrm{~nm}$ bandwidth, the TE and TM polarization extinction ratios are higher than $18 \mathrm{~dB}$ and $30 \mathrm{~dB}$, respectively. Compared with the previous polarization beam splitters, our structure is simple, the size is small, and the extinction ratio is high, which meets the needs of modern optical communications, optical interconnection, and optical integrated systems.
\end{abstract}

Keywords: photonic crystal; polarization beam splitter; self-collimation effect; photonic bandgap

\section{Introduction}

A polarization beam splitter (PBS) refers to a beam splitter that can separate different polarization modes in electromagnetic waves and propagate in different directions. It is widely used in optical communications, optical storage, and optical integration systems. The traditional PBS uses the different refractive index of polarized light in the crystal. Its size is generally on the order of millimeters, since it mainly depends on natural crystal materials. Because it is susceptible to angles and the extinction ratio of transmitted light is not high, it is challenging to use PBS to meet the needs of modern optical integrated devices.

The concept of photonic crystals was first proposed in 1987 when E. Yablonoviceton [1] studied the radiation characteristics of materials. He believed that the suppression of spontaneous emission could be achieved by changing the photon density of states. In the same year, S. John [2,3] studied the photonic localization based on disordered artificial materials. He proposed that changing the photonic crystal structure can control the beam propagation characteristics. The photonic crystal is a kind of man-made material [4], which is a combination of dielectric materials with different dielectric constants in space according to a specific periodic law. It has the characteristics of photonic bandgap [5,6] and photonic localization [7-9]. Based on this characteristic, photonic crystals are widely used in various photonic devices, such as photonic bandgap [10,11], filters [12], beam splitters [13-15], switches [16], multiplexers [17], and modulators [18-20]. Photonic crystal fibers (PCFs) and polymer optical fibers (POFs) are often used in multiplexers, demultiplexers, combiners, 
beam splitters, and other applications [21-23]. The combination of materials and photonic crystal structures improves system performance and reduces power consumption. It has great potential to be integrated into dense wavelength division multiplexing technology to improve network system performance.

The combination of photonic crystals with polarization multiplexing technology can be used to design photonic crystal polarization beam splitters [24-26]. According to the bandgap characteristics, negative refraction characteristics [27], self-collimation effect [28], multi-mode interference theory, the directional coupling effect, guided-mode dispersion relationship, and other photonic crystal characteristics [29], the propagation of electromagnetic waves in the photonic crystal can be controlled. The following is the pioneering works of several photonic crystal polarizers. The earliest photonic crystal polarization beam splitter is a heterojunction structure prepared on SOI, using the selfcollimation effect of polarized light in different materials. But the transmittance of the two polarized lights is not high [30]. Polarization beam splitting can be achieved by inserting a material with high reflectivity for a polarized light into the self-collimating structure. Such a polarization mode is effective at suppressing diffraction consumption [31]. The polarization beam splitter can also be prepared by inserting air holes on the silicon plate, and the high extinction ratio of the two polarized lights can be obtained [32].

In recent years, there have been more developments in the structural design and research of photonic crystal polarization beam splitters. In 2005, Yogita Kalra [33] designed an ultra-compact polarization beam splitter based on a complete photonic bandgap. By digging out two rows of linear defect waveguides in a photonic crystal with a honeycomb structure, the TE mode and TM mode are directed along different waveguides. This structure that uses defect structures to achieve polarization beam splitting is relatively simple, but the polarization extinction is relatively small. In 2016, Siqi Duan [27] used lithium niobate $(\mathrm{LN})$ materials and the negative refractive photonic crystal wedge plate with an angle of $60^{\circ}$ to propose an efficient broadband polarization beam splitter. The polarization beam splitter based on LN has a transmittance of more than $80 \%$ for polarized lights. The angle and wavelength bandwidth at $1550 \mathrm{~nm}$ is $8^{\circ}$ and $70 \mathrm{~nm}$, respectively. In this kind of polarization beam splitting realized by using negative refraction materials, the application field is limited and the light easily diverges at the exit end. In 2017, Haiyang Wang [29] used dual-core photonic crystal fiber and magnetic fluid to propose a tunable surface plasmon resonance based on a polarization beam splitter. When the external magnetic field is in the range of 400-760 Oe, the wavelength of the incident light can be separated in the same fiber within a wide range of $1.45-1.55 \mu \mathrm{m}$. A high extinction ratio and wide bandwidth can be obtained under different light wavelengths. This structure that uses an external magnetic field to control polarization beam splitting is complicated and difficult to manufacture.

Based on the above problems, a new photonic crystal polarization beam splitter based on the self-collimation effect and photonic bandgap characteristics was designed. Different from other published polarization beam splitters that change the filling ratio, the device in this paper achieved polarization separation by changing the hole shape, that is, rectangular holes were used to replace the traditional circular holes. The overall structure was a combination of a rectangular holes structure and circular holes structure to obtain 90 degree separation of TE and TM polarized lights. The device had a simple structure, a small size, and a high polarization extinction ratio.

\section{Structure Design}

\subsection{Self-Collimating Structure}

When defects and nonlinear materials are not inserted in the photonic crystal, the light beam irradiates the surface of the photonic crystal, and an abnormal dispersion phenomenons appear inside the photonic crystal, that is, the self-collimation effect of the photonic crystal [10], and the light beam do not appear in the photonic crystal. The effects of reflection, refraction, diffraction, etc., always propagate in a straight line inside the 
photonic crystal. The self-collimating structure designed based on this characteristic is shown in Figure 1a. We inserted $21 \times 21$ circular holes arranged in a square lattice, where the distance between the hole and the adjacent hole was the lattice constant $a(a=0.43$ im), and the radius of the holes was $r(r=0.33 \mathrm{a})$. After optimizing the parameters of this structure, a better self-collimation effect could be obtained. The energy band diagram of the self-collimating structure was calculated by Plane-wave Expansion (PWE), as shown in Figure $1 \mathrm{~b}$. It can be seen from the figure that TE polarization and TM polarization have no photonic bandgap in the normalized frequency range of $0-0.7 \mathrm{a} / \lambda$ (corresponding to the wavelength range of $\lambda>614 \mathrm{~nm}$ ). Optical signals within the frequency range of the photonic bandgap cannot propagate in the photonic crystal structure. Therefore, both TE polarization and TM polarization can pass through this structure.

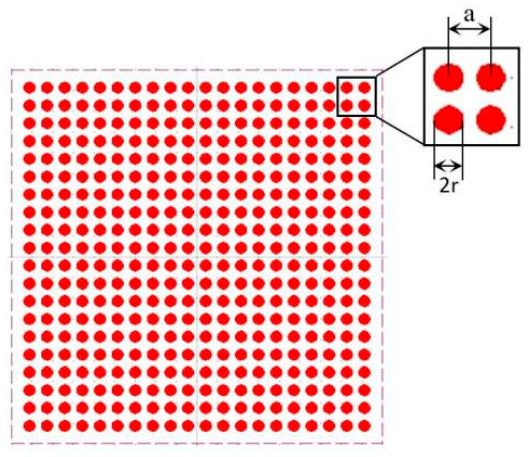

(a)

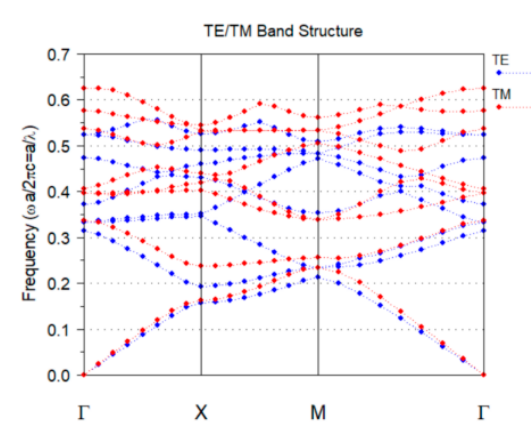

(b)

Figure 1. (a) Self-collimating structure; (b) Energy band diagram of self-collimating structure.

In order to further research the transmission behavior of TE polarized light and TM polarized light in the structure, the equifrequency contours (EFC) of the first Brillouin zone were calculated by PWE. EFC is a series of cross-sections of the full-band surface at different frequencies. The propagation of light in the photonic crystal is controlled by the dispersion surface of the photonic crystal, and the group velocity determines the propagation direction, so it is always perpendicular to the EFC. If the EFC remains flat at a particular frequency, self-collimation will occur. The EFC diagrams corresponding to the second energy band in TE and TM modes are shown in Figure 2a,b, respectively. The number of the second energy band in Figure $1 \mathrm{~b}$ is 1 , so the upper part of Figure 2 was labeled "band 1". It can be seen from the figure that in the normalized frequency range $0.27-0.28 \mathrm{a} / \lambda$, the EFC in TE and TM modes is almost linearly distributed near the $\mathrm{k}_{\mathrm{x}}$ direction and $\mathrm{k}_{\mathrm{z}}$ direction, which shows that both TE polarized light and TM polarized light can propagate in a self-collimated manner in the structure along the $\mathrm{k}_{\mathrm{x}}$ direction and the $\mathrm{k}_{\mathrm{z}}$ direction. We chose the center position below the self-collimating structure as the input terminal and placed a continuous wave light source with a Gaussian time distribution with a length of 4 lattice parameters. We then placed the power monitor (to detect the power at the output terminal) in the lower center of the structure as the signal output terminal. Taking into account the reflection on the surface of the photonic crystal, we set the excitation source closer to the lower input end. The electromagnetic field distribution at different times in this self-collimating structure was calculated by the Finite Difference Time Domain (FDTD) method. Figure $3 a, b$ respectively show the wavelength at $1550 \mathrm{~nm}$ (The normalized frequency is $0.276 \mathrm{a} / \lambda$.) of the field distribution of TE polarized light and TM polarized light in the self-collimating structure. There was still partial reflection (blue part) at the lower input end of Figure 5a,b, but both TE polarized light and TM polarized light could be well coupled into the self-collimating structure. It could be observed that both polarized lights can be effectively self-collimated and propagate along the $\mathrm{Z}$ direction. 


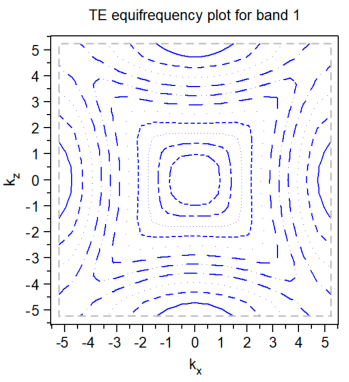

(a)

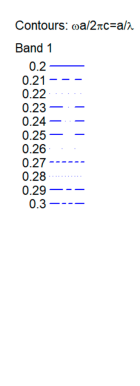

Figure 2. (a) EFC diagram in TE mode; (b) EFC diagram in TM mode.

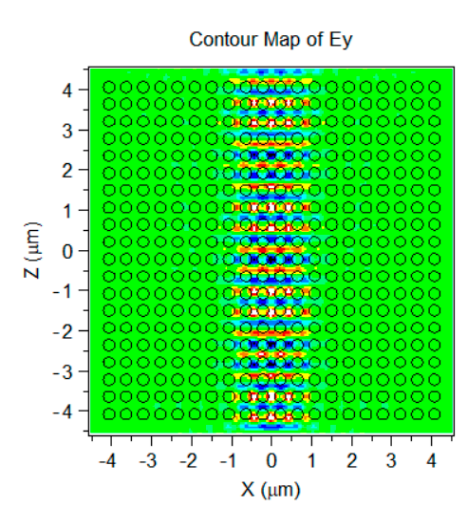

(a)
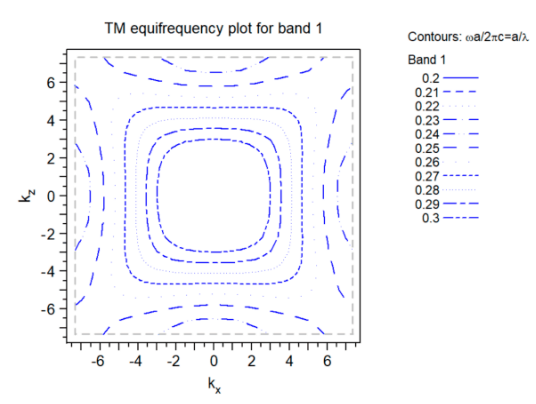

(b)
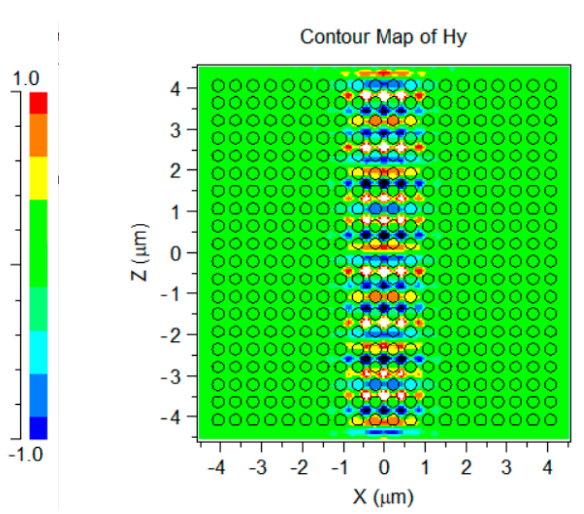

(b)

Figure 3. (a) EFC diagram in TE mode; (b) EFC diagram in TM mode.

\subsection{Polarization Beam Splitting Structure}

The photonic bandgap is one of the characteristics of photonic crystals. Due to the periodic distribution of the dielectric material of the photonic crystal in space, there may be a photonic bandgap between adjacent energy bands. When the frequency of electromagnetic waves falls within the frequency range of the photonic bandgap, the electromagnetic wave will not propagate in this photonic crystal.

We used this feature to design a polarization beam splitting structure, as shown in Figure 4a. In order to ensure the consistency of the lattice constant of the entire device, the form of inserting air holes in the silicon background material was still selected. The type of air hole inserted was a rectangular hole. The length of the four sides of a rectangular hole was the same, which was defined as $d$, the values of which were twice the radius $r$ of the circular hole. Figure $4 \mathrm{~b}$ is the energy band diagram in TE mode and TM mode obtained by calculating the polarization beam splitting structure according to the PWE algorithm. It can be seen that the energy band in TE mode has no photonic bandgap, which means that TE polarized light can be passing directly through the polarization beam splitting structure; while in the TM mode, there is a photonic bandgap (red region) with a normalized frequency in the range of $0.247-0.291 \mathrm{a} / \lambda$ (corresponding to the wavelength range of 1477.7-1740.9 nm). This means TM polarized light in this frequency range cannot pass through the structure. 


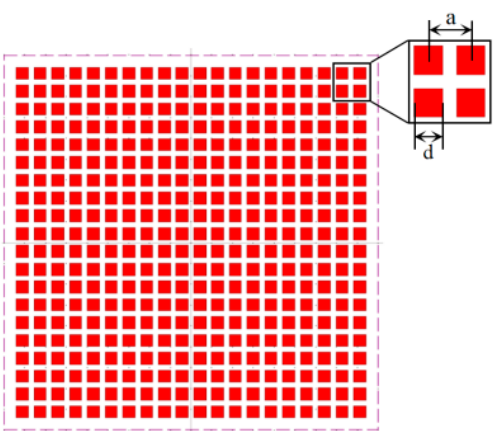

(a)

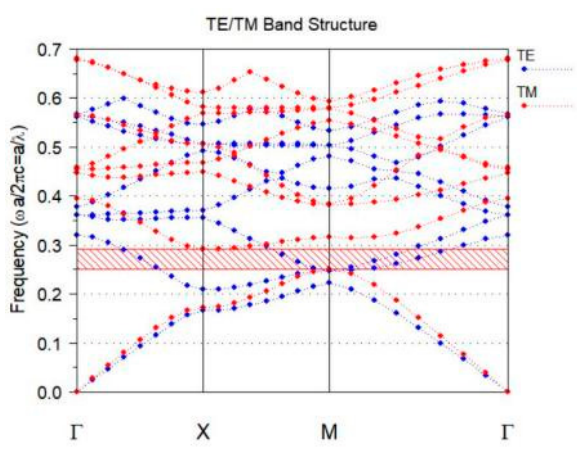

(b)

Figure 4. (a) Polarization beam splitting structure; (b) Energy band diagram of polarization beam splitting structure.

The EFC diagram in TE mode is shown in Figure 5. The normalized frequency range is $0.27-0.28 \mathrm{a} / \lambda$ (corresponding to the wavelength range of $1535.7-1592.6 \mathrm{~nm}$ ). Because the EFC in TE mode is linearly distributed along the $k_{x}$ and $k_{z}$ directions, TE polarized light can propagate in the structure along the $\mathrm{k}_{\mathrm{x}}$ and $\mathrm{k}_{\mathrm{z}}$ directions in a self-collimating manner. However, TM polarized light at $1550 \mathrm{~nm}$ cannot pass through this structure because its frequency is within the photonic bandgap, thus realizing the function of polarization beam splitting. We chose the center position below the self-collimating structure as the input terminal and placed a continuous light source there with a Gaussian time distribution and a length of 4 lattice parameters. The power monitor was placed in the center above the self-collimating structure as the output terminal. Taking into account the reflection on the surface of the photonic crystal, we set the excitation source closer to the lower input end. The TE polarized light and TM polarized light at a wavelength of $1550 \mathrm{~nm}$ were set to match the boundary conditions according to the FDTD algorithm perfectly, and the obtained steady-state field distribution diagrams are shown in Figure 6a,b. From the bottom of Figure 6a, it can be seen that there is still some reflection (blue part) at the input end, but most of the light is directly coupled into the polarization beam splitting structure. In Figure 6b, because TM polarized light cannot be coupled in, almost all TM polarized light is reflected back to the input end. It can be observed that the TE mode can propagate effectively in the self-collimating structure along the positive z-axis, but the TM mode cannot enter the polarization beam splitting structure and is reflected at the input end.

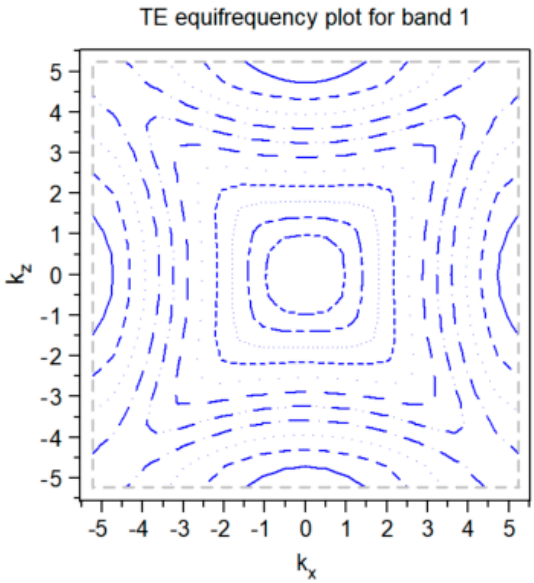

$$
\begin{aligned}
& \text { Contours: } \omega \mathrm{a} / 2 \pi \mathrm{c}=\mathrm{a} / \lambda \\
& \text { Band } 1 \\
& 0.2- \\
& 0.21--- \\
& 0.22 \\
& 0.23-- \\
& 0.24-- \\
& 0.25-- \\
& 0.26 \\
& 0.27 \\
& 0.28 \\
& 0.29--- \\
& 0.3---
\end{aligned}
$$

Figure 5. EFC diagram in TE mode. 


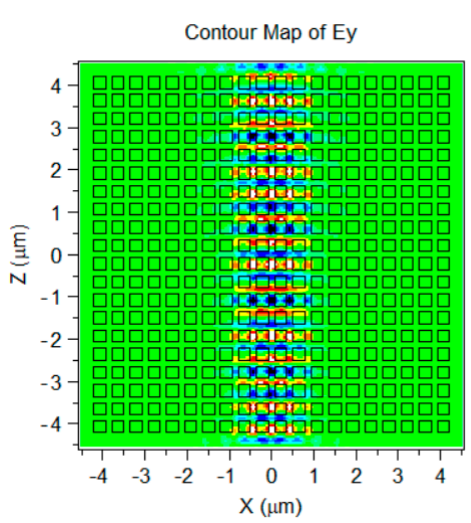

(a)
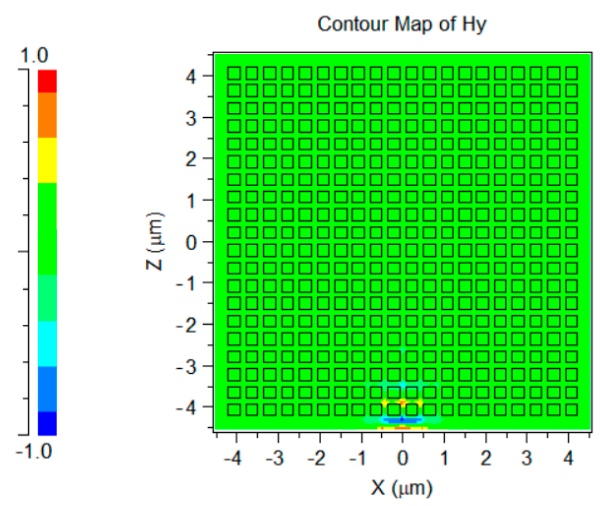

(b)

Figure 6. (a) Steady-state field distribution in TE mode; (b) Steady-state field distribution in TM mode.

\section{Compact Polarization Beam Splitter}

To achieve the orthogonal separation of TE polarized light and TM polarized light, we combined the designed self-collimation structure and polarization beam splitting structure. As shown in Figure 7, the polarized beam splitting part was inserted into the hypotenuse of the self-collimating part. In the diagonal area, the number of sides of the inserted polarization beam splitting part is $n$, as shown in Figure 7, where $n$ is 5 . The entire device is still $21 \times 21$ air columns inserted into the background material $\mathrm{Si}$, the background refractive index is 3.4 , and the air column refractive index is 1 . Because the side length $\mathrm{d}$ of the square air column in the polarization beam splitting structure is the same as the diameter of the circular air column in the self-collimating structure, the lattice constant of the entire device is the same, which is still $0.43 \mu \mathrm{m}$.

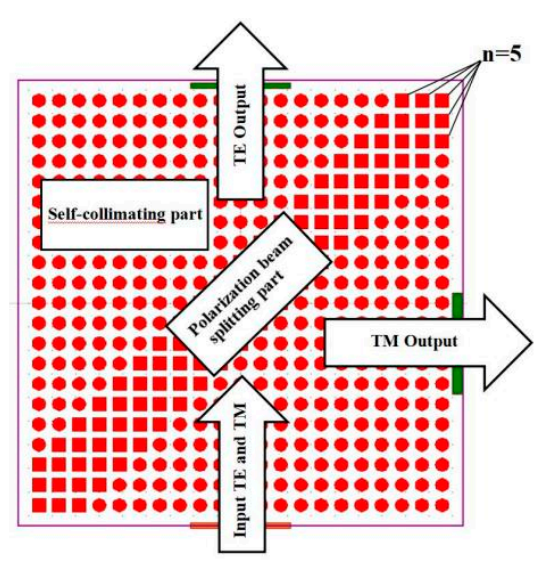

Figure 7. Compact polarization beam splitter structure.

The input end of the entire device, that is, the excitation source, was set at the center of the lower end. The TE output end was set at the center of the upper end of the device, and the TM output end moved up and down in the z-axis direction according to the inserted polarization beam splitting structure to ensure that the center axis of the output end was aligned with the input end. The center axis of the excitation source was symmetrical about the right hypotenuse, and a power monitor was placed at the output end. TE polarized light and TM polarized light were input from the input end and propagated upwards along with the self-collimating structure. When the TM polarized light encountered the polarization beam splitting structure, it could not pass through the structure and was reflected to the right end (the TM output). The TE polarized light was directly transmitted through the polarization beam splitting structure and output from the TE output terminal, thereby realizing the orthogonal separation of TE polarized light and TM polarized light. 
The number of sides $\mathrm{n}$ of the polarization splitting structure inserted in the left hypotenuse area of the compact polarization beam splitter had little effect on the transmittance of TE polarized light, and mainly had a relatively significant influence on the transmittance of TM polarized light. We mainly researched the influence on TE transmittance and TM transmittance when $\mathrm{n}$ was $1,3,5$, and 7 . When $\mathrm{n}$ was set to 1 , both TE polarized light and TM polarized light were output from the TE output end, while the transmittance was more than $90 \%$, and the reflection effect on TM was not realized; when $n$ was set to 3, part of the TM polarized light was reflected into the TM output terminal, but there was still a part of TM polarized light output from the TE output terminal through the polarization beam splitting structure. At this time, the transmittance of the TM output terminal was less than $70 \%$.

When $n$ was 5 or 7 , TM could be perfectly reflected to the TM output end to achieve the orthogonal separation of TE polarized light and TM polarized light. Considering the surface reflection and internal scattering of the photonic crystal, Figure 8a,b respectively show the steady-state field distribution of two polarized lights at $1550 \mathrm{~nm}$ in the polarization beam splitter. It can be seen from the stable field that although there are still reflected electromagnetic waves (blue parts) at the input end, most of the input light is transmitted from the input end. TE polarized light propagates in a self-collimating structure, and almost no deflection occurs at the interface between the self-collimating structure and the polarization beam splitting structure. We then continued to propagate along the $\mathrm{z}$ direction, as shown in Figure 8a, and finally output from the TE output. The TM polarized light also propagated along the $+z$ direction in the self-collimating structure. But it was then reflected 90 degrees to the right side of the device at the interface between the selfcollimating structure and the polarization beam splitting structure. Finally, it propagated in the positive direction of $x$ and was output from the TM output terminal, as shown in the Figure $8 \mathrm{~b}$. Power monitors were placed at the TE output terminal and TM output terminal to calculate the optical power when passing through the center of the monitor. When $\mathrm{n}$ was 5, the transmittance of TE polarized light at the TE output end was 95.4\%, and the transmittance of TM output end was $0.54 \%$; the transmittance of TM polarized light at the TM output end was $88.63 \%$, and the transmittance of TE output end was $0.023 \%$. The reflection on the surface of the photonic crystal structure and the scattering inside the photonic crystal had little effect on the propagation of TE polarized light in the device, and the transmittance of TE polarized light was greater than 95\%. The TM polarized light was greatly affected by reflection at the junction of the polarization beam splitting structure and the self-collimating structure, resulting in the transmittance of TM being lower than 90\%. The reflection at the input end hardly affected the transmittance of the two polarized lights.

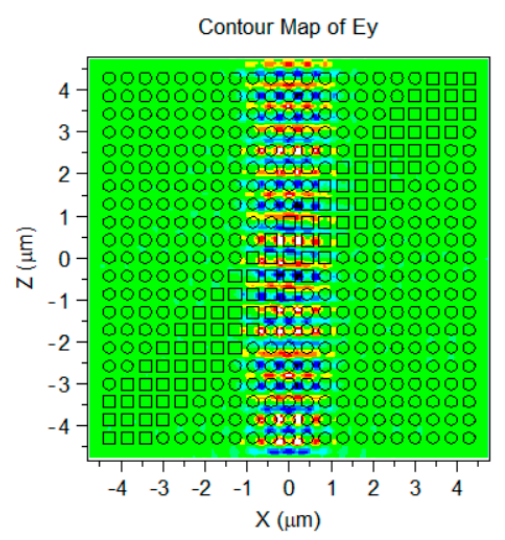

(a)

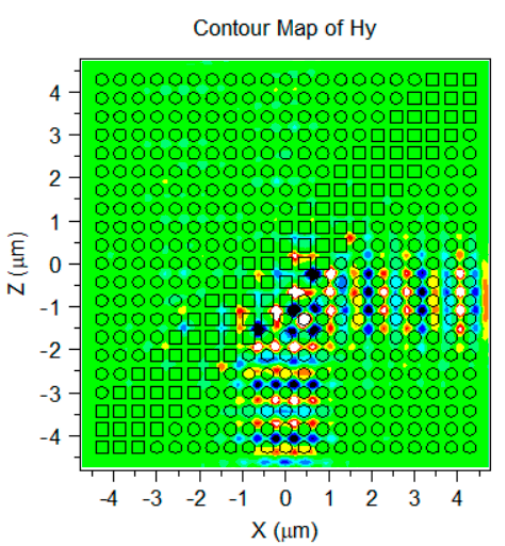

(b)

Figure 8. At $1550 \mathrm{~nm}$, when $\mathrm{n}$ is 5 (a) steady-state field distribution in TE mode; (b) steady-state field distribution in TM mode. 
In order to eliminate the influence of the boundary on the performance of the device, the boundary condition of the Perfectly Matched Layer (PML) was selected. The PML absorbs all incident energy without producing reflections. This allowed field energy which is incident on the boundary to effectively leave the domain. Using the FDTD algorithm, the transmission spectrum can be calculated in the wavelength range of $1500-1600 \mathrm{~nm}$ (the corresponding normalized frequency range is $0.267-0.285 \mathrm{a} / \lambda$ ). For TE polarized light, the transmission spectra of the TE output end and the TM output end are shown in Figure 9a. In the normalized collimation frequency range of $0.267-0.285 \mathrm{a} / \lambda($ or $\lambda=1500-1600 \mathrm{~nm})$, the transmittance of the TM output terminal remained almost zero, while the transmittance of the TE output terminal increased from $78 \%$ to $97.8 \%$, with TE at $1555 \mathrm{~nm}$. The maximum transmittance was $97.8 \%$. The TM transmittance was almost zero because TM polarized light was reflected to the right when passing through the polarization beam splitting structure, and almost no TM light passed through the upper monitor. The reason for the increase in TE transmittance is related to the wavelength. From the previous EFC diagram, it can be seen that as the wavelength increases, the EFC in TE mode tends to be straight, the self-collimation effect improves, and TE polarized light extends further. It is output from the upper TE output terminal in the $\mathrm{z}$ direction. Figure $9 \mathrm{~b}$ shows the transmission spectrum of TM polarized light. In the wavelength range of $1500 \mathrm{~nm}-1600 \mathrm{~nm}$, the transmittance of the TM output port exhibited waveform fluctuations, with several peaks that appeared, and the peak range was mostly between $80-95 \%$. The reason for the fluctuation of the TM transmittance may be that the inserted polarization beam splitting structure changed the propagation path of the TM polarized light, which led to a change in the self-collimation direction. A series of dispersions such as the scattering and refraction of the polarized light itself also caused the fluctuation of the power. From the previous EFC diagram in the TM mode, the self-collimation effect tended to be better as the wavelength increased. Based on the superposition of the two, it is reasonable that the TM transmittance fluctuates as the wavelength increases. At $\lambda=1550 \mathrm{~nm}, \mathrm{TE}(\mathrm{TM})$ polarization had a high transmittance of 95.4\% (88.5\%) through the TE(TM) output terminal.

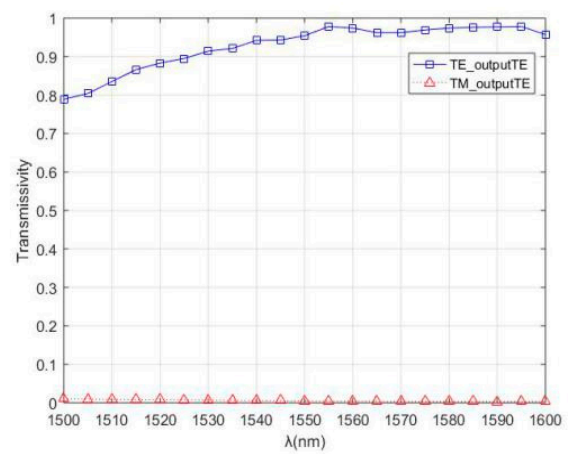

(a)

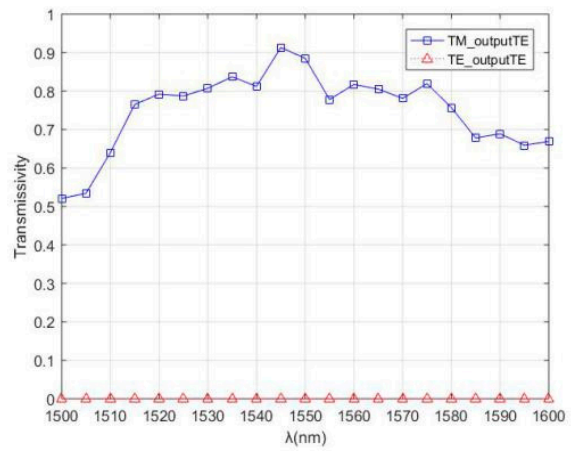

(b)

Figure 9. When $\mathrm{n}$ is 5, the transmission spectrum of (a) TE polarized light and (b) TM polarized light at the output end.

In addition to transmittance, an essential indicator for measuring polarization beam splitters is the Polarization Extinction Ratio (PER), which can measure the separation of two polarized lights. In the device structure, the PER of TE polarization and TM polarization can be expressed by the following formula:

$$
\begin{aligned}
& P E R_{T E}=10 \log \left(T_{1-T E} / T_{1-T M}\right), \\
& P E R_{T M}=10 \log \left(T_{2-T M} / T_{2-T E}\right),
\end{aligned}
$$

where $\mathrm{T}_{1-\mathrm{TE}}$ and $\mathrm{T}_{1-\mathrm{TM}}$ refer to the TE transmittance and TM transmittance at the TE output end, $\mathrm{T}_{2-\mathrm{TM}}$ and $\mathrm{T}_{2-\mathrm{TE}}$ refer to the TM transmittance and TE transmittance at the TM output end, and this unit is dB. The PER calculated according to Figure 9 is shown in 
Figure 10. In the wavelength range of $1500-1600 \mathrm{~nm}$ (100 nm bandwidth), PERTM and PERTE exceeded $30 \mathrm{~dB}$ and $18 \mathrm{~dB}$, respectively, and the maximum values reached $37 \mathrm{~dB}$ and $25 \mathrm{~dB}$. At a wavelength of $1550 \mathrm{~nm}, \mathrm{PER}_{\mathrm{TE}}\left(\mathrm{PERT}_{\mathrm{M}}\right)$ was $23 \mathrm{~dB}(37 \mathrm{~dB})$, which indicated that TE (TM) polarized light has a high PER of $23 \mathrm{~dB}(37 \mathrm{~dB})$.

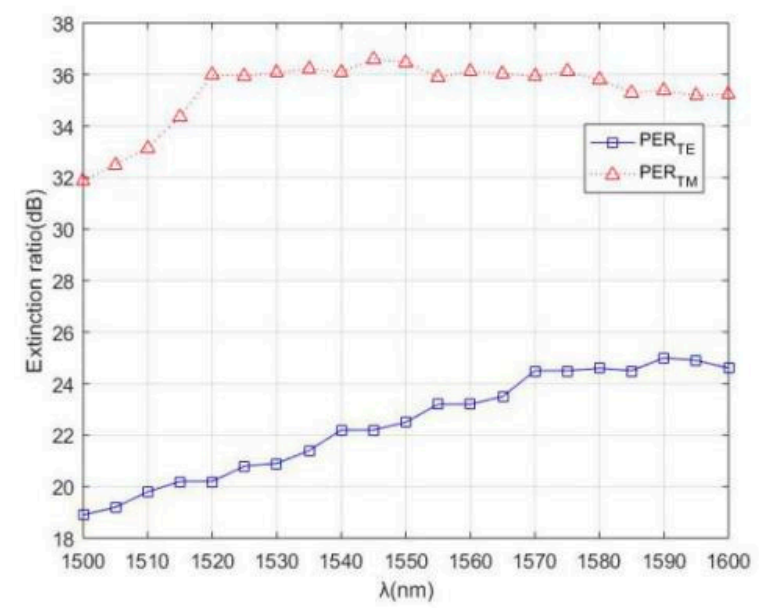

Figure 10. PER of outputs TE and TM over the band $\ddot{\mathrm{e}}=1500-1600 \mathrm{~nm}$.

\section{Discussions}

Compared with the polarization beam splitter designed by changing the radius of the circular hole, the polarization beam splitting structure we designed uses a square hole structure. However, in terms of preparation, the difficulty of preparing square holes is slightly higher than that of circular holes. However, the polarization structure of different apertures has the problem that the lattice period does not match the larger aperture. As a result, the lattice period must be increased to ensure that the circular holes do not overlap each other. This will increase the size of the device. Therefore, compared with the old polarization beam splitters with different circular hole radii, the square hole structure proposed in this paper will reduce the size of the entire device. It can be seen from the previous results that, compared with the circular hole structure, the transmittance and extinction ratio of the device we designed at TM and TE polarized light were improved. Therefore, the square hole structure we proposed provides a new design idea for the photonic crystal polarization beam splitter, which is more suitable for photonic integrated devices.

Compared with the extinction ratios of TE polarized light and TM polarized light of $18 \mathrm{~dB}$ and $23 \mathrm{~dB}$ in reference [20], the polarization extinction ratio of the compact device we designed was increased by $5 \mathrm{~dB}$ and $4 \mathrm{~dB}$, respectively, and the transmittance of TM was also higher. In terms of preparation difficulty, rectangular holes are indeed difficult to use to guarantee absolute right angles, but right angles have little effect on device parameters. Compared with the preparation of round holes, the preparation of rectangular holes is also more difficult, but it is easier than the preparation of other hole types. As long as the horizontal and vertical dimensions are the same as the diameter of the circular hole, and the TE polarization and TM polarization can be separated by 90 degrees, the high polarization extinction ratio of the device can be achieved.

\section{Conclusions}

A new type of compact two-dimensional photonic crystal polarization beam splitter is proposed according to the self-collimation effect and photonic bandgap characteristics of the two-dimensional photonic crystal. The device is composed of a rectangular air hole type polarization beam splitting structure and a circular air hole type self-collimating structure. By inserting the polarization structure of the rectangular hole row into the hypotenuse area of the self-collimating structure arranged in a square lattice, the orthogonal separation of TE and TM polarized light can be achieved. The change of TE (TM) transmittance with 
the number of rectangular hole rows in the inserted polarization beam splitter structure was analyzed and discussed. When the number of rectangular hole rows is 5 and the wavelength is $1550 \mathrm{~nm}$, the transmittance of TE polarized light at the TE output end is $95.4 \%$, the transmittance of TM output end is $0.54 \%$, and the polarization extinction ratio of TE is $23 \mathrm{~dB}$; TM polarized light is at the TM output end. The transmittance is $88.63 \%$, the transmittance at the TE output end is $0.023 \%$, and the polarization extinction ratio of TM is $37 \mathrm{~dB}$. Within the wavelength range of $1500-1600 \mathrm{~nm}$ (100 $\mathrm{nm}$ bandwidth), PER $\mathrm{TM}_{\mathrm{TM}}$ and PER $\mathrm{TE}_{\mathrm{T}}$ exceed $30 \mathrm{~dB}$ and $15 \mathrm{~dB}$, respectively, with a maximum of $37 \mathrm{~dB}$ and $25 \mathrm{~dB}$, respectively. This compact polarization beam splitter enables the orthogonal separation of TE polarized light and TM polarized light. Compared with some devices that have been published, the structure is simpler, and the size is smaller. The polarization extinction ratio is higher, which can better meet the needs of modern optical integration devices. The device works at $1550 \mathrm{~nm}$, has a working bandwidth of up to $100 \mathrm{~nm}$, has high transmittance and high polarization extinction ratio in the range of $1500-1600 \mathrm{~nm}$, and has a large application space in optical interconnection systems and optical communication systems. It can be used as a input signal polarization processor for multiplexers and demultiplexers. It can also be used as a input signal polarization processor for multiplexers and demultiplexers. The device size is $9 \mu \mathrm{m} \times 9 \mu \mathrm{m}$, which is a compact photonic device suitable for optical integrated systems.

Author Contributions: Writing—original draft preparation, G.T.; writing—review and editing, G.T., H.H. and Y.L.; Conceptualization and methodology, G.T. and H.W.; Supervision and Revision, H.W. All authors have read and agreed to the published version of the manuscript.

Funding: This work was supported by the Science and Technology Plan of Guangdong Province (Nos.2017B010112003, 2020B010171001), and Guangzhou Municipal Science and Technology Plan (Nos. 2016,04046021,201905010001), and Science and Technology Development Plan of Zhongshan City (Nos. 2019AG014, 2019AG042, 2020AG023).

Informed Consent Statement: Informed consent was obtained from all subjects involved in the study.

Conflicts of Interest: The authors declare no conflict of interest.

\section{References}

1. Yablonovich, E. Inhibited spontaneous emission in solid-state phsics and electronics. Phys. Rev. Lett. 1987, 58, 2059-2063. [CrossRef] [PubMed]

2. John, S. Strong Localization of Photons in Certain Disordered Dielectric Super Lattices. Phys. Rev. Lett. 1987, 58, 2486-2489. [CrossRef]

3. Busch, K.; John, S. Photonic bandgap formation in certain self-organizing systems. J. Lightwave Technol. 1998, 17, 1931-1943.

4. Yablonovitch, E.; Gmitter, T.J.; Leung, K.M. Photonic Band Structure: The Face-Centered-Cubic Case Employing Nonspherical Atoms. Phys. Rev. Lett. 1991, 67, 2295-2298. [CrossRef] [PubMed]

5. Satoshi, I.; Yasutomo, O.; Yasuhiko, A. Recent progress in topological waveguides and nanocavities in a semiconductor photonic crystal platform. Phys. Rev. Lett. 2021, 11, 319-337.

6. Wang, L.H.; Yang, S.H. Nano photoelectric material structures-Photonic crystals. Adv. Mater. Res. 2013, 677, 9-15. [CrossRef]

7. Risa, S.; Takahiro, I.; Yasushi, T. Utilizing Broadband Light from a Superluminescent Diode for Excitation of Photonic Crystal High-Q Nanocavities. J. Lightwave Technol. 2019, 37, 2458-2466.

8. Nurul, A.; Yuta, O.; Tomohisa, $\mathrm{T}$. $\mathrm{SiO}_{2}$ clad active and passive photonic crystal nanocavity devices fabricated with photolithography: Toward future mass production of photonic crystal nanocavity devices. In Proceedings of the 2017 Conference on Lasers and Electro-Optics Pacific Rim, Singapore, 31 July-4 August 2017; pp. 1-2.

9. Nassibeh, E.; Reza, Y.; Hamed, B. Ultrahuge Light Intensity in the Gap Region of a Bowtie Nanoantenna Coupled to a Low-modevolume Photonic-crystal Nanocavity. Curr. Opt. Photonics 2018, 2, 85-89.

10. Sayed, E.; Israa, A.; Karim, K. Five-Line Photonic Crystal Waveguide for Optical Buffering and Data Interconnection of Picosecond Pulse. J. Lightwave Technol. 2019, 37, 788-798.

11. Adam, M. Symmetry-engineered waveguide dispersion in PT symmetric photonic crystal waveguides. J. Opt. Soc. Am. 2020, B37, 168-180.

12. Wang, J.; Jiang, S.; Jia, L. Research on the phase-shifted fiber grating spectrum characteristics in the inhomogeneous strain fields. Spectrosc. Spectr. Anal. 2012, 32, 2003-2008.

13. Pavicic, M. Spin-correlated interferometry for polarized and unpolarized photons on a beam splitter. Phys. Rev. At. Mol. Opt. Phys. 1994, 50, 3486-3490. [CrossRef] 
14. Luhua, X.; Yun, W.; Deng, M. Compact Broadband Polarization Beam Splitter Based on Multimode Interference Coupler with Internal Photonic Crystal for the SOI Platform. J. Lightwave Technol. 2019, 37, 1231-1240.

15. Yasa, U.G.; Turduev, M.; Giden, I.H.; Kurt, H. Extinction Ratio Polarization Beam Splitter Design by Low-Symmetric Photonic Crystals. J. Lightwave Technol. 2017, 35, 1677-1683. [CrossRef]

16. Deeksha, R.; Rajinder, S.K.; Balveer, P. All-optical NOR gate based on cross structures in 2D photonic crystal using logic NOT and OR gates. J. Opt. Technol. 2017, 84, 851-857.

17. Yoshino, M.; Miki, N.; Yoshimoto, N. Multiwavelength optical source for OCDM using sinusoidally modulated laser diode. J. Lightwave Technol. 2009, 27, 4524-4529. [CrossRef]

18. Zhang, X.; Chung, C.J.; Hosseini, A.; Subbaraman, H.; Luo, J.; Jen, A.K.; Nelson, R.L.; Lee, C.Y.; Chen, R.T. High Performance Optical Modulator Based on Electro-Optic Polymer Filled Silicon Slot Photonic Crystal Waveguide. J. Lightwave Technol. 2016, 34, 2941-2951. [CrossRef]

19. Huang, Y.; Wang, Y.; Zhang, L.; Shao, Y.; Zhang, F.; Liao, C.; Wang, Y. Tunable Electro-Optical Modulator Based on a Photonic Crystal Fiber Selectively Filled with Liquid Crystal. J. Lightwave Technol. 2019, 37, 1903-1908. [CrossRef]

20. Yosuke, T.; Tomoki, T.; Yosuke, H. Si Photonic Crystal Slow-Light Modulators with Periodic p-n Junctions. J. Lightwave Technol. 2017, 35, 1684-1692.

21. Bar, G.; Linoy, A.; Dror, M. RGB wavelength multiplexer based on polycarbonate multicore polymer optical fiber. Opt. Fiber Technol. 2021, 61, 102441.

22. Dror, M.; Gilad, K. An Eight-Channel C-Band Demux Based on Multicore Photonic Crystal Fiber. Nanomaterials $2018,8,845$.

23. Dror, M.; Aaron, P. Power splitting of $1 \times 16$ in multicore photonic crystal fibers. Nanomaterials 2017, 417, 34-39.

24. Lei, X.Y.; Li, H.; Ding, F. Novel application of a perturbed photonic crystal: High-quality filter. Appl. Phys. Lett. 1997, 71, 2889-2891. [CrossRef]

25. Young, A.B.; Thijssen, A.C.; Beggs, D.M. Polarization engineering in photonic crystal waveguides for spin-photon entanglers. Phys. Rev. Lett. 2015, 115, 153901. [CrossRef] [PubMed]

26. Liu, X.; Galfsky, T.; Sun, Z. Strong light-matter coupling in two-dimensional atomic crystals. Nat. Photonics 2015, 9, 30-32. [CrossRef]

27. Duan, S.; Chen, Y.; Li, G. Broadband polarization beam splitter based on a negative refractive lithium niobate photonic crystal slab. Chin. Opt. Lett. 2016, 14, 042301. [CrossRef]

28. Xing, L.; Lingtao, M.; Chao, W. Tunable beam propagating characteristics of two-dimensional rectangular-rod photonic crystals based on self-collimation effect. Opt. Commun. 2018, 427, 311-318.

29. Wang, H.; Yan, X.; Li, S.; Zhang, X. Tunable surface plasmon resonance polarization beam splitter based on dual-core photonic crystal fiber with magnetic fluid. Opt. Quantum Electron. 2017, 49, 368. [CrossRef]

30. Schonbrun, E.; Wu, Q.; Park, W.; Yamashita, T.; Summers, C.J. Polarization beam splitter based on a photonic crystal heterostructure. Opt. Lett. 2006, 31, 3104. [CrossRef]

31. Zabelin, V.; Dunbar, L.A.; Le Thomas, N.; Houdré, R.; Kotlyar, M.V.; O’Faolain, L.; Krauss, T.F. Self-collimating photonic crystal polarization beam splitter. Opt. Lett. 2007, 32, 530. [CrossRef]

32. Park, J.M.; Lee, S.G.; Park, H.R.; Lee, M.H. High-efficiency polarization beam splitter based on a self-collimating photonic crystal. Opt. Soc. Am. 2010, 27, 2247. [CrossRef]

33. Kalra, Y.; Sinha, R.K. Design of Ultra Compact Polarization Splitter on the Complete Photonic Band Gap. Opt. Quantum Electron. 2005, 37, 889-895. [CrossRef] 